BNL-67630

August 15, 2000

\title{
Measurement of $\mathbf{B}\left(K^{+} \rightarrow \pi^{+} \nu \bar{\nu}\right)^{1}$
}

\author{
S.H. Kettell ${ }^{2}$ \\ Brookhaven National Laboratory
}

\begin{abstract}
The experimental measurement of $K^{+} \rightarrow \pi^{+} \nu \bar{\nu}$ is reviewed. New results from experiment E787 at BNL are presented: with data from 1995-97 the branching ratio has been measured to be $\mathrm{B}\left(K^{+} \rightarrow \pi^{+} \nu \bar{\nu}\right)=\left(1.5_{-1.2}^{+3.4}\right) \times 10^{-10}$. The future prospects for additional data in this mode are examined.
\end{abstract}

\section{INTRODUCTION}

The unprecedented sensitivities of rare kaon decay experiments and the recent observation of $K^{+} \rightarrow \pi^{+} \nu \bar{\nu}$ have opened doors to the measurement of the unitarity triangle completely within the kaon system. The decay $K^{+} \rightarrow \pi^{+} \nu \bar{\nu}$ is one of the 'golden modes' for measuring CKM parameters. Measurement of the branching ratio $\mathrm{B}\left(K^{+} \rightarrow \pi^{+} \nu \bar{\nu}\right)$ provides a clean and unambiguous determination of the CKM matrix element $\left|V_{t d}\right|$, in particular of the quantity $\left|\lambda_{t}\right| \equiv\left|V_{t s}^{*} V_{t d}\right|$.

The theoretical uncertainty in $K^{+} \rightarrow \pi^{+} \nu \bar{\nu}$ is quite small $(\sim 7 \%)$ as the hadronic matrix element is extracted from the $K \rightarrow \pi e \nu_{e}$ branching ratio $\mathrm{B}\left(K_{e 3}\right)$. The $K^{+} \rightarrow \pi^{+} \nu \bar{\nu}$ branching ratio has been calculated to next-to-leading-log approximation [1], with isospin violation corrections [2] and two-loop-electroweak effects [3]. The branching ratio can be expressed as [4]

$$
\begin{aligned}
B\left(K^{+} \rightarrow \pi^{+} \nu \bar{\nu}\right) & =\frac{\kappa_{+} \alpha^{2} B\left(K_{e 3}\right)}{2 \pi^{2} \sin ^{4} \theta_{W}\left|V_{u s}\right|^{2}} \sum_{l}\left|X_{t} \lambda_{t}+X_{c} \lambda_{c}\right|^{2} \\
& =8.88 \times 10^{-11} A^{4}\left[\left(\bar{\rho}_{0}-\bar{\rho}\right)^{2}+(\sigma \bar{\eta})^{2}\right] \\
& =3.6 \times 10^{-4}\left(\left[\operatorname{Re}\left(\lambda_{t}\right)-1.4 \times 10^{-4}\right]^{2}+\left[\operatorname{Im}\left(\lambda_{t}\right)\right]^{2}\right) \\
& =(8.2 \pm 3.2) \times 10^{-11},
\end{aligned}
$$

with the error determined by the present uncertainty of $\lambda_{t}$. The current limit on $B_{s}-\bar{B}_{s}$ mixing, combined with the measured frequency of $B_{d}-\bar{B}_{d}$ mixing,

1) To be published in the Proceedings of the th $^{\text {th }}$ Conference on the Intersections of Particle and Nuclear Physics; Quebec City, Canada, May 22-28, 2000; Z. Parsa and W. Marciano, Eds.

2) for the E787 collaboration 
implies a limit on $K^{+} \rightarrow \pi^{+} \nu \bar{\nu}$ that has very small theoretical uncertainty [4]: $\mathrm{B}\left(K^{+} \rightarrow \pi^{+} \nu \bar{\nu}\right)<1.67 \times 10^{-10}$.

\section{EXPERIMENT E787}

The E787 experiment at BNL [5] was designed to search for $K^{+} \rightarrow \pi^{+} \nu \bar{\nu}$ and collected data in 1989-91 [6] and then again in 1995-98 after an upgrade to the detector and beamline [7].

The first observation of $K^{+} \rightarrow \pi^{+} \nu \bar{\nu}$ was reported in the analysis of the 1995 data set [8]. A new and improved analysis of the 1995-97 data set has reduced the background levels by almost a factor of three. A plot of range vs. energy for events passing all other $K^{+} \rightarrow \pi^{+} \nu \bar{\nu}$ criteria is shown in Figure 1(a). One event

a)

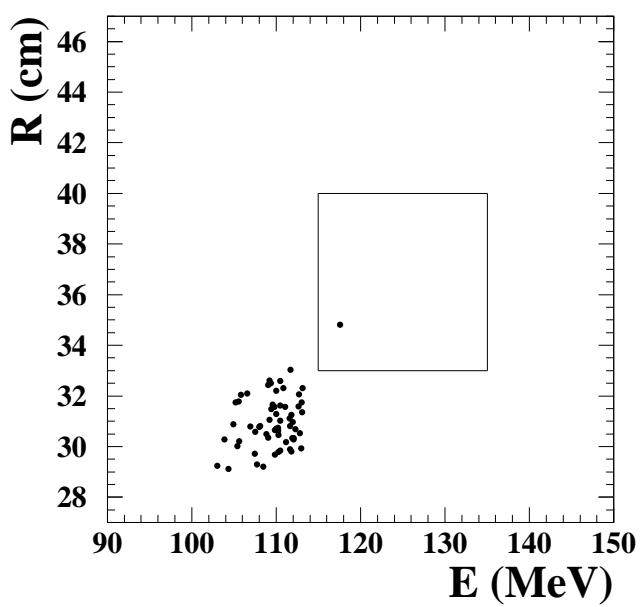

b)

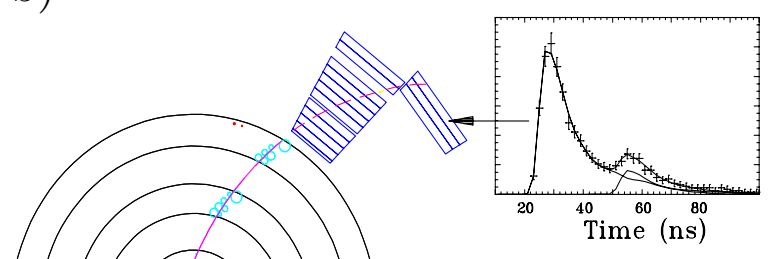

FIGURE 1. (a) Range vs. Kinetic energy plot of the final sample. The events near $E=108 \mathrm{MeV}$ are $K_{\pi 2}$ background. The box indicates the accepted region for $K^{+} \rightarrow \pi^{+} \nu \bar{\nu}$ events. (b) Event display of the $K^{+} \rightarrow \pi^{+} \nu \bar{\nu}$ signal event.

is observed in the signal region (the same one as observed in the 1995 data: see Ref. 8). A reconstruction of the event is shown in Figure 1(b). The background is measured to be $0.08 \pm 0.02$ events. Based on this one event, the branching ratio is $\mathrm{B}\left(K^{+} \rightarrow \pi^{+} \nu \bar{\nu}\right)=\left(1.5_{-1.2}^{+3.4}\right) \times 10^{-10}[9]$. From this measurement, a limit of

$$
0.002<\left|V_{t d}\right|<0.04
$$

is determined; in addition, the following limits on $\lambda_{t} \equiv V_{t s}^{*} V_{t d}$ can be set:

$$
\begin{aligned}
\left|\operatorname{Im}\left(\lambda_{t}\right)\right| & <1.22 \times 10^{-3} \\
-1.10 \times 10^{-3}<\operatorname{Re}\left(\lambda_{t}\right) & <1.39 \times 10^{-3} \\
1.07 \times 10^{-4}<\quad\left|\lambda_{t}\right| & <1.39 \times 10^{-3} .
\end{aligned}
$$


The final sensitivity of the E787 experiment, based on data from 1995-98, should reach a factor of two further to the standard-model (SM) expectation for $K^{+} \rightarrow \pi^{+} \nu \bar{\nu}$.

The $90 \%$ CL upper limit on $K^{+} \rightarrow \pi^{+} \nu \bar{\nu}$ is $\mathrm{B}\left(K^{+} \rightarrow \pi^{+} \nu \bar{\nu}\right)<5.8 \times 10^{-10}$ and a model-independent limit [10] on the neutral mode, $K_{L}^{\circ} \rightarrow \pi^{\circ} \nu \bar{\nu}$, can be derived from this result:

$$
\begin{aligned}
B\left(K_{L}^{\circ} \rightarrow \pi^{\circ} \nu \bar{\nu}\right) & <4.4 \times B\left(K^{+} \rightarrow \pi^{+} \nu \bar{\nu}\right) \\
& <2.6 \times 10^{-9}(90 \% \mathrm{CL})
\end{aligned}
$$

The $K \rightarrow \pi \pi \nu \bar{\nu}$ decays, such as $K^{+} \rightarrow \pi^{+} \pi^{\circ} \nu \bar{\nu}$, can also, in principle, provide a clean determination of CKM matrix parameters; however, due to the small SM branching ratios, e.g. $\mathrm{B}\left(K^{+} \rightarrow \pi^{+} \pi^{\circ} \nu \bar{\nu}\right)=(1-2) \times 10^{-14}$, their usefulness is limited. E787 has recently set the first limit on any of these modes [11], with $\mathrm{B}\left(K^{+} \rightarrow \pi^{+} \pi^{\circ} \nu \bar{\nu}\right)<4.3 \times 10^{-5}$. Additional new results from E787 include a measurement of the direct emission (DE) radiation in $K^{+} \rightarrow \pi^{+} \pi^{\circ} \gamma$ decay $\left(\mathrm{K}_{\pi 2 \gamma}\right)$ and the first observation of structure-dependent radiation (SD) in $K^{+} \rightarrow \mu^{+} \nu_{\mu} \gamma$ decays $\left(\mathrm{K}_{\mu 2 \gamma}\right)$. With eight times higher statistical sensitivity than previous experiments and better kinematic constraints E787 has measured a branching ratio for direct emission $\mathrm{B}\left(\mathrm{K}_{\pi 2 \gamma}: \mathrm{DE}\right)$ that is four times smaller than previous results [12], $\mathrm{B}\left(\mathrm{K}_{\pi 2 \gamma}: \mathrm{DE}, 55 \mathrm{MeV}<T_{+}<90 \mathrm{MeV}\right)=(4.7 \pm 0.8 \pm 0.3) \times 10^{-6}\left(T_{+}\right.$is the kinetic energy of the $\pi^{+}$). E787 has also measured the branching ratio for structuredependent $\mathrm{K}_{\mu 2 \gamma}[13]$ to be $\mathrm{B}\left(\mathrm{K}_{\mu 2 \gamma}: S D^{+}\right)=(1.33 \pm 0.12 \pm 0.18) \times 10^{-5}$. The vector and axial-vector form factors are $\left|F_{V}+F_{A}\right|=0.165 \pm 0.007 \pm 0.011$ and $-0.04<F_{V}-F_{A}<0.24$ at $90 \%$ CL.

\section{FUTURE PROSPECTS}

Significant progress in the determination of the fundamental CKM parameters from the $K \rightarrow \pi \nu \bar{\nu}$ system will be made in the generation of experiments that is now starting: E949 and KOPIO at BNL, CKM and KAMI at FNAL and E391 at KEK. These measurements can unambiguously determine SM $C P$ violation parameters. Comparison with the B-system will then over-constrain the triangle and test the SM explanation of $C P$ violation. The two most important tests are expected to be:

- Comparison of the angle $2 \beta$ from the ratio $\mathrm{B}\left(K^{+} \rightarrow \pi^{+} \nu \bar{\nu}\right) / \mathrm{B}\left(K_{L}^{\circ} \rightarrow \pi^{\circ} \nu \bar{\nu}\right)$ and the $C P$ asymmetry in the decay $B_{d}^{\circ} \rightarrow \psi K_{S}^{\circ}[10,14]$.

- Comparison of the magnitude $\left|V_{t d}\right|$ from $K^{+} \rightarrow \pi^{+} \nu \bar{\nu}$ and the ratio of the mixing frequencies of $B_{s}$ and $B_{d}$ mesons [4].

Improvement in the charged mode $K^{+} \rightarrow \pi^{+} \nu \bar{\nu}$ will come in two steps: E949 and CKM.

The E787 experiment has already demonstrated sufficient background rejection for a very precise measurement of $\mathrm{B}\left(K^{+} \rightarrow \pi^{+} \nu \bar{\nu}\right)$. A new experiment under construction, E949, is expected to run in 2001-03. Taking advantage of the very large 
AGS proton flux and the experience gained with the E787 detector, E949 with modest upgrades should observe $\mathcal{O}(10) \mathrm{SM}$ events in a two year run. The background is well-understood and is $\sim 10 \%$ of the SM signal.

A proposal for a further factor of 10 improvement has been initiated at FNAL. The CKM experiment (E905) plans to collect $\mathcal{O}(100)$ SM events, with $\mathcal{O}(10)$ background events, in a two year run starting after 2005. This experiment will use a new technique, with $\mathrm{K}^{+}$decay-in-flight and independent momentum (Si and straw-tube trackers) and velocity (kaon and pion RICH) spectrometers. CKM is situated in a high flux $22 \mathrm{GeV} / \mathrm{c}$ RF-separated kaon beam, derived from the Main Injector at FNAL.

Improvements in the sensitivities of $K^{+} \rightarrow \pi^{+} \nu \bar{\nu}$ experiments over time is shown in Figure 2. Published data are shown as solid points and future projections to

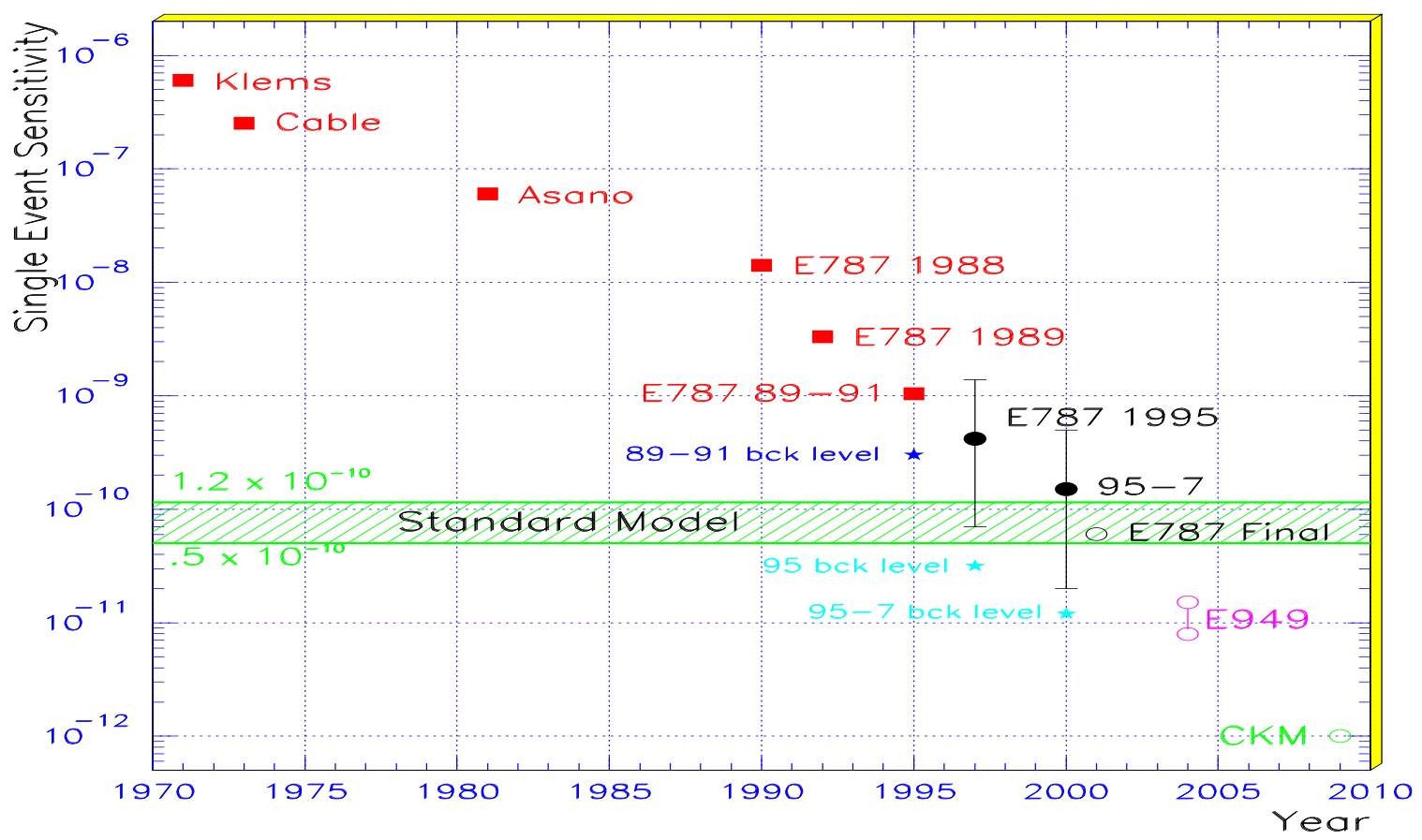

FIGURE 2. Sensitivities of experiments searching for $K^{+} \rightarrow \pi^{+} \nu \bar{\nu}$. The sensitivities of experiments setting limits are shown as red squares. The projected sensitivities are shown as open circles. Background measurements for several of the recent E787 searches are shown as blue stars.

E949 and CKM are shown as open points.

\section{ACKNOWLEDGMENTS}

This work was supported under U.S. Department of Energy contract \#DE-AC0298CH10886. 


\section{REFERENCES}

1. Bucahalla G. and Buras A., Nucl. Phys. B412, 106 (1994).

2. Marciano W.J. and Parsa Z., Phys. Rev. D53, R1 (1996).

3. Bucahalla G. and Buras A., Phys. Rev. D57, 216 (1998).

4. Bucahalla G. and Buras A., Nucl. Phys. B548, 309 (1999).

5. Atiya M., et al., Nucl. Instrum. Methods A321, 129 (1992); Atiya M., et al., Nucl. Instrum. Methods A279, 180 (1989).

6. Adler S., et al., Phys. Rev. Lett. 76, 1421 (1996).

7. Chiang I.H., et al., IEEE Trans. Nucl. Sci. NS-42, 394 (1995); Bryman D.A., et al., Nucl. Instrum. Methods A396, 394 (1997); Blackmore E.W., et al., Nucl. Instrum. Methods A404, 295 (1998); Komatsubara T.K., et al., Nucl. Instrum. Methods A404, 315 (1998); Doornbos J. et al., Nucl. Instrum. Methods A444, 546 (2000).

8. Adler S., et al., Phys. Rev. Lett. 79, 2204 (1997).

9. Adler S., et al., Phys. Rev. Lett. 84, 3768 (2000).

10. Grossman Y., and Nir Y., Phys. Lett. B398, 163 (1997).

11. Ng C. The Search for the Rare Decay of $K^{+} \rightarrow \pi^{+} \pi^{\circ} \nu \bar{\nu}$. PhD thesis. SUNY Stonybrook (2000); also submitted to Phys. Rev. D.

12. Adler S., et al., Phys. Rev. Lett. in press; also hep-ex/0007021.

13. Adler S., et al., Phys. Rev. Lett. in press; also hep-ex/0003019..

14. Bucahalla G. and Buras A., Phys. Lett. B333, 221 (1994); Bucahalla G. and Buras A., Phys. Rev. D54, 6782 (1996); Nir Y. and Worah M.P., Phys. Lett. B423, 319 (1998); Bergmann S. and Perez G., hep-ph/0007170. 